\title{
ERRATUM
}

\section{Erratum for Rattenbacher et al., Analysis of CUGBP1 Targets Identifies GU-Repeat Sequences That Mediate Rapid mRNA Decay}

Bernd Rattenbacher, ${ }^{1,2}$ Daniel Beisang, ${ }^{1,2}$ Darin L. Wiesner, ${ }^{1,2}$ Jonathan C. Jeschke, ${ }^{1,2}$ Maximilian von Hohenberg, ${ }^{1,2}$ Irina A. Vlasova-St. Louis, ${ }^{1,2}$ Paul R. Bohjanen ${ }^{1,2,3}$

Center for Infectious Diseases and Microbiology Translational Research, ${ }^{1}$ Department of Microbiology, ${ }^{2}$ and Department of Medicine, ${ }^{3}$ University of Minnesota, Minneapolis, Minnesota, USA

Volume 30, no. 16, p. 3970-3980, 2010. Page 3970: The article byline should read as given above. 